Supporting Information for

\title{
Large variations in hygroscopic properties of unconventional mineral dust
}

Chao Peng, Wenjun Gu, Rui Li, Qinhao Lin, Qingxin Ma, Shiguo Jia, Padmaja Krishnan,Xinming Wang, and Mingjin Tang

Correspondence: Mingjin Tang (mingjintang@gig.ac.cn)

The SI includes 7 pages, 2 tables and 4 figures. 
Table S1. Integrated areas of IR peaks assigned to adsorbed water as a function of RH for nine fly ash samples. Wave number ranges used for integration are $3646-2800 \mathrm{~cm}^{-1}$ for BCR-176R, 3700$2700 \mathrm{~cm}^{-1}$ for BCR-615, 3649-2600 $\mathrm{cm}^{-1}$ for CFA-2689, 3631-2700 $\mathrm{cm}^{-1}$ for CFA-2690, 3679$2800 \mathrm{~cm}^{-1}$ for CFA-2691, 3700-2800 $\mathrm{cm}^{-1}$ for GBW08401, 3700-2700 $\mathrm{cm}^{-1}$ for GBW11128a, $3700-2500 \mathrm{~cm}^{-1}$ for GBW11129a and 3629-2800 $\mathrm{cm}^{-1}$ for GBW11131a.

\begin{tabular}{|c|c|c|c|c|c|}
\hline $\mathrm{RH}(\%)$ & peak area & $\mathrm{RH}(\%$ & peak area & $\mathrm{RH}(\%)$ & peak area \\
\hline \multicolumn{2}{|c|}{ BCR-176R } & \multicolumn{2}{|c|}{ BCR-615 } & \multicolumn{2}{|c|}{ CFA-2689 } \\
\hline$<1$ & 0 & $<1$ & 0 & $<1$ & 0 \\
\hline 20 & 1.47 & 20 & 9.95 & 18 & 0.34 \\
\hline 40 & 3.83 & 39 & 294.79 & 37 & 0.73 \\
\hline 60 & 24.46 & 57 & 410.33 & 57 & 1.86 \\
\hline 78 & 57.91 & 74 & 497.46 & 73 & 4.89 \\
\hline 89 & 117.31 & 83 & 551.73 & 83 & 6.56 \\
\hline \multicolumn{2}{|c|}{ CFA-2690 } & \multicolumn{2}{|c|}{ CFA-2691 } & \multicolumn{2}{|c|}{ GBW08401 } \\
\hline$<1$ & 0 & $<1$ & 0 & $<1$ & 0 \\
\hline 20 & 0.09 & 18 & 0.40 & 20 & 0.11 \\
\hline 40 & 0.20 & 39 & 0.68 & 40 & 0.16 \\
\hline 59 & 0.43 & 58 & 0.98 & 60 & 0.22 \\
\hline 77 & 1.07 & 74 & 1.44 & 76 & 0.33 \\
\hline 84 & 1.70 & 85 & 1.86 & 85 & 0.44 \\
\hline \multicolumn{2}{|c|}{ GBW11128a } & \multicolumn{2}{|c|}{ GBW11129a } & \multicolumn{2}{|c|}{ GBW11131a } \\
\hline$<1$ & 0 & $<1$ & 0 & $<1$ & 0 \\
\hline 19 & 0.81 & 20 & 0.51 & 19 & 0.37 \\
\hline 39 & 1.00 & 40 & 0.76 & 39 & 0.63 \\
\hline 59 & 1.69 & 60 & 1.27 & 59 & 0.81 \\
\hline 75 & 2.50 & 76 & 1.76 & 78 & 1.15 \\
\hline 82 & 3.01 & 86 & 2.21 & 86 & 1.62 \\
\hline
\end{tabular}


Table S2. Mass ratios of adsorbed water to the dry sample $\left(m_{\mathrm{w}} / m_{0}\right)$ as a function of RH $(0-90 \%)$ at $25^{\circ} \mathrm{C}$ for all samples investigated in this work. All the errors given are standard deviations.

\begin{tabular}{|c|c|c|c|c|c|c|}
\hline \multirow{2}{*}{$\begin{array}{l}\mathrm{RH} \\
(\%)\end{array}$} & \multicolumn{6}{|c|}{$m_{\mathrm{w}} / m_{0}\left(\times 10^{-3}\right)$} \\
\hline & BCR-176R & BCR-615 & OFA & UPM1648a & CFA-2689 & CFA-2690 \\
\hline 10 & $3.9 \pm 0.1$ & $7.0 \pm 0.1$ & $5.6 \pm 0.1$ & $4.1 \pm 0.1$ & $0.3 \pm 0.1$ & $0.2 \pm 0.1$ \\
\hline 20 & $7.1 \pm 0.1$ & $32.3 \pm 1.0$ & $10.4 \pm 0.1$ & $6.9 \pm 0.1$ & $0.5 \pm 0.1$ & $0.3 \pm 0.1$ \\
\hline 30 & $9.8 \pm 0.2$ & $48.1 \pm 1.5$ & $24.7 \pm 1.0$ & $9.5 \pm 0.1$ & $0.6 \pm 0.1$ & $0.5 \pm 0.1$ \\
\hline 40 & $12.5 \pm 0.2$ & $64.2 \pm 2.0$ & $46.2 \pm 0.7$ & $12.6 \pm 0.1$ & $0.7 \pm 0.1$ & $0.7 \pm 0.1$ \\
\hline 50 & $15.9 \pm 0.2$ & $83.7 \pm 2.6$ & $66.1 \pm 0.9$ & $16.7 \pm 0.1$ & $0.9 \pm 0.1$ & $0.8 \pm 0.1$ \\
\hline 60 & $21.6 \pm 0.2$ & $120.3 \pm 3.8$ & $90.9 \pm 1.2$ & $22.6 \pm 0.1$ & $1.0 \pm 0.1$ & $0.9 \pm 0.1$ \\
\hline 70 & $91.8 \pm 1.7$ & $279.3 \pm 1.7$ & $123.8 \pm 1.6$ & $32.4 \pm 0.1$ & $1.1 \pm 0.1$ & $1.0 \pm 0.1$ \\
\hline 80 & $327.1 \pm 0.2$ & $399.4 \pm 2.6$ & $173.1 \pm 2.1$ & $56.0 \pm 0.1$ & $1.2 \pm 0.1$ & $1.1 \pm 0.1$ \\
\hline \multirow[t]{2}{*}{90} & $629.6 \pm 1.9$ & $734.0 \pm 3.0$ & $272.1 \pm 3.0$ & $183.5 \pm 0.1$ & $1.5 \pm 0.1$ & $1.2 \pm 0.1$ \\
\hline & CFA-2691 & GBW08401 & GBW11128a & GBW11129a & GBW11131a & \\
\hline 10 & $0.3 \pm 0.1$ & $0.4 \pm 0.1$ & $0.3 \pm 0.1$ & $0.1 \pm 0.1$ & $0.1 \pm 0.1$ & \\
\hline 20 & $0.4 \pm 0.1$ & $0.7 \pm 0.1$ & $0.4 \pm 0.1$ & $0.2 \pm 0.1$ & $0.1 \pm 0.1$ & \\
\hline 30 & $0.5 \pm 0.1$ & $1.0 \pm 0.1$ & $0.6 \pm 0.1$ & $0.2 \pm 0.1$ & $0.1 \pm 0.1$ & \\
\hline 40 & $0.5 \pm 0.1$ & $1.3 \pm 0.1$ & $0.7 \pm 0.1$ & $0.3 \pm 0.1$ & $0.2 \pm 0.1$ & \\
\hline 50 & $0.6 \pm 0.1$ & $1.7 \pm 0.1$ & $0.9 \pm 0.1$ & $0.3 \pm 0.1$ & $0.2 \pm 0.1$ & \\
\hline 60 & $0.7 \pm 0.1$ & $1.9 \pm 0.1$ & $1.1 \pm 0.1$ & $0.3 \pm 0.1$ & $0.2 \pm 0.1$ & \\
\hline 70 & $0.7 \pm 0.1$ & $2.1 \pm 0.1$ & $1.3 \pm 0.1$ & $0.4 \pm 0.1$ & $0.2 \pm 0.1$ & \\
\hline 80 & $0.8 \pm 0.1$ & $2.4 \pm 0.1$ & $1.6 \pm 0.1$ & $0.4 \pm 0.1$ & $0.3 \pm 0.1$ & \\
\hline 90 & $1.0 \pm 0.1$ & $3.0 \pm 0.1$ & $2.7 \pm 0.1$ & $0.6 \pm 0.1$ & $0.3 \pm 0.1$ & \\
\hline
\end{tabular}



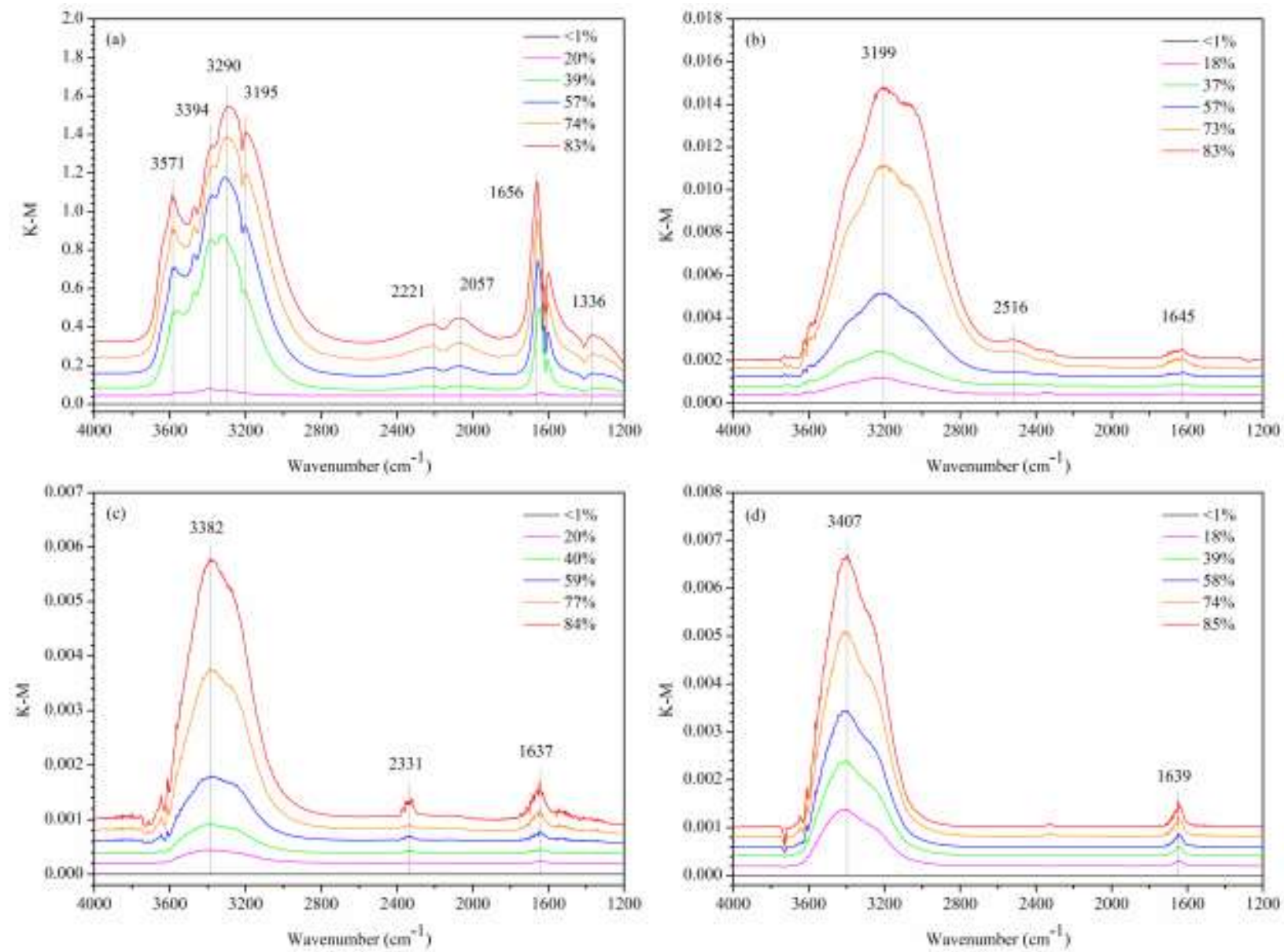

Figure S1. In situ DRIFTS spectra as a function of RH (0-90\%) at $25{ }^{\circ} \mathrm{C}$ for (a) BCR-615, (b) CFA-2689, (c) CFA-2690, (d) CFA-2691. 

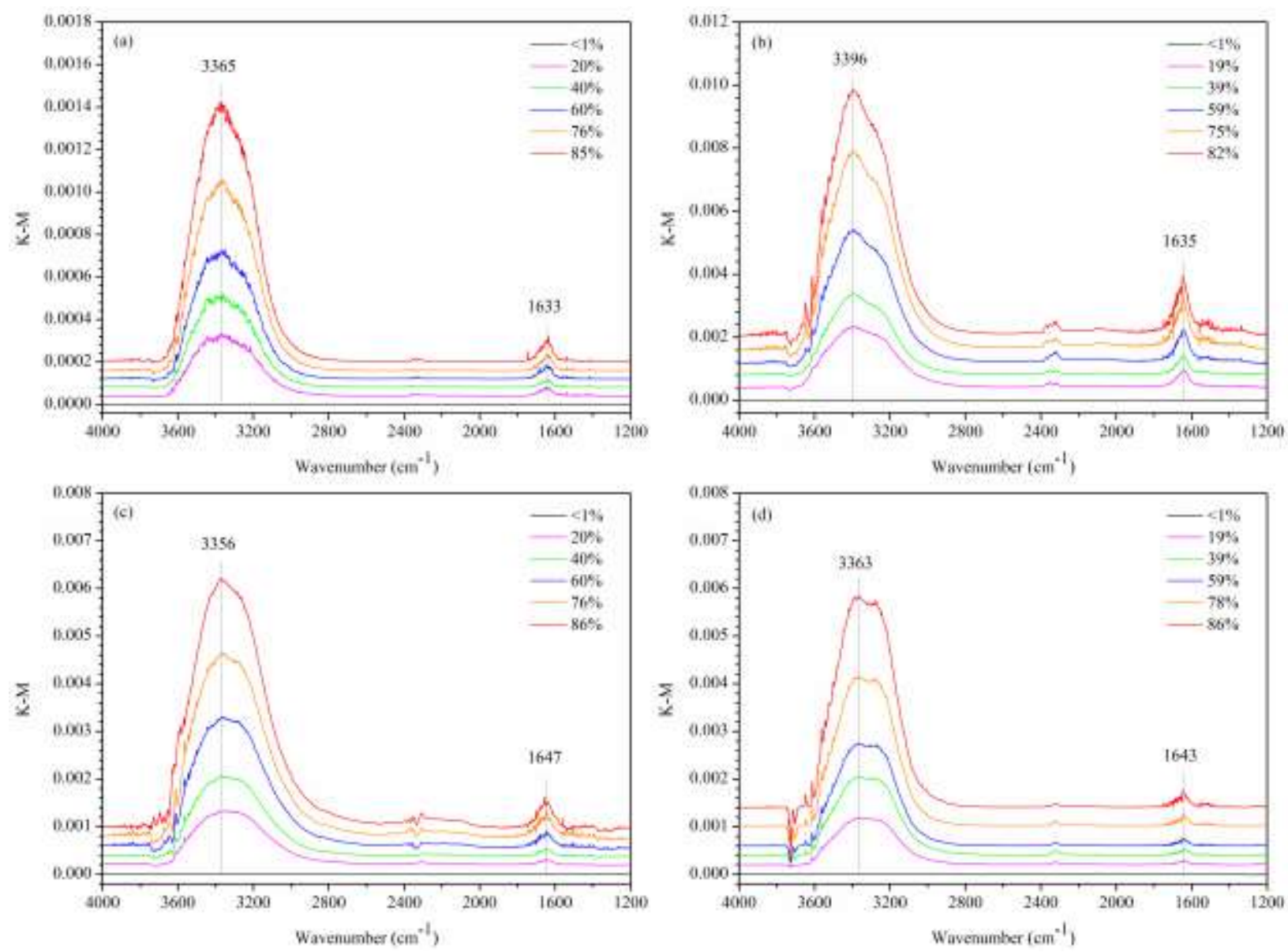

Figure S2. In situ DRIFTS spectra as a function of RH (0-90\%) at $25^{\circ} \mathrm{C}$ for (a) GBW08401, (b) GBW11128a, (c) GBW11129a and (d) GBW11131a. 

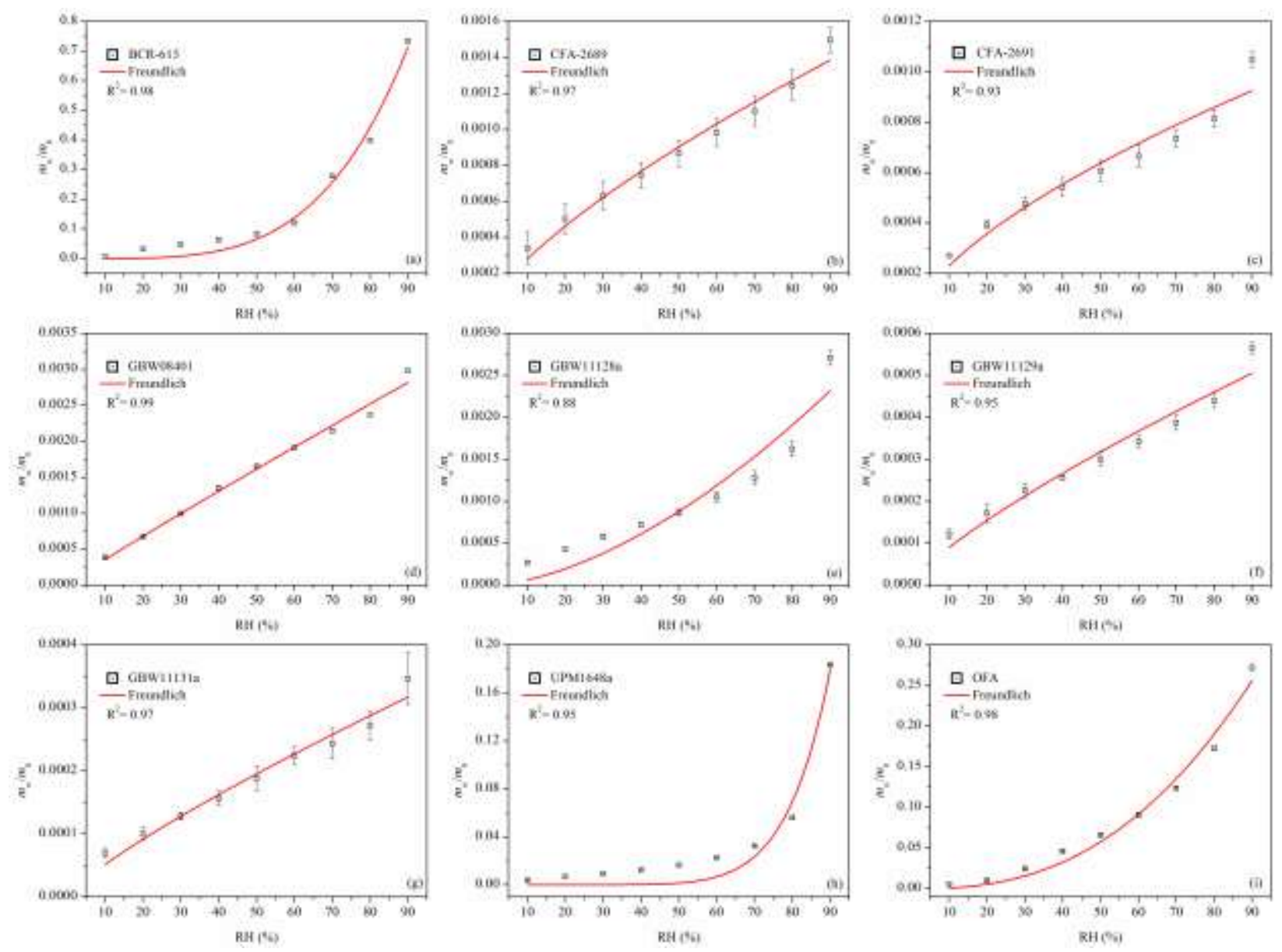

Figure S3. Mass ratios of adsorbed water to dry samples $\left(m_{\mathrm{w}} / m_{0}\right)$ as a function of RH $(0-90 \%)$ at $25^{\circ} \mathrm{C}$ for (a) BCR-615, (b) CFA-2689; (c) CFA-2691; (d) GBW08401; (e) GBW11128a; (f) GBW11129a; (g) GBW11131a; (h) UPM1648a and (i) OFA. The experimental data were fitted with Freundlich adsorption isotherm model (solid curves). 

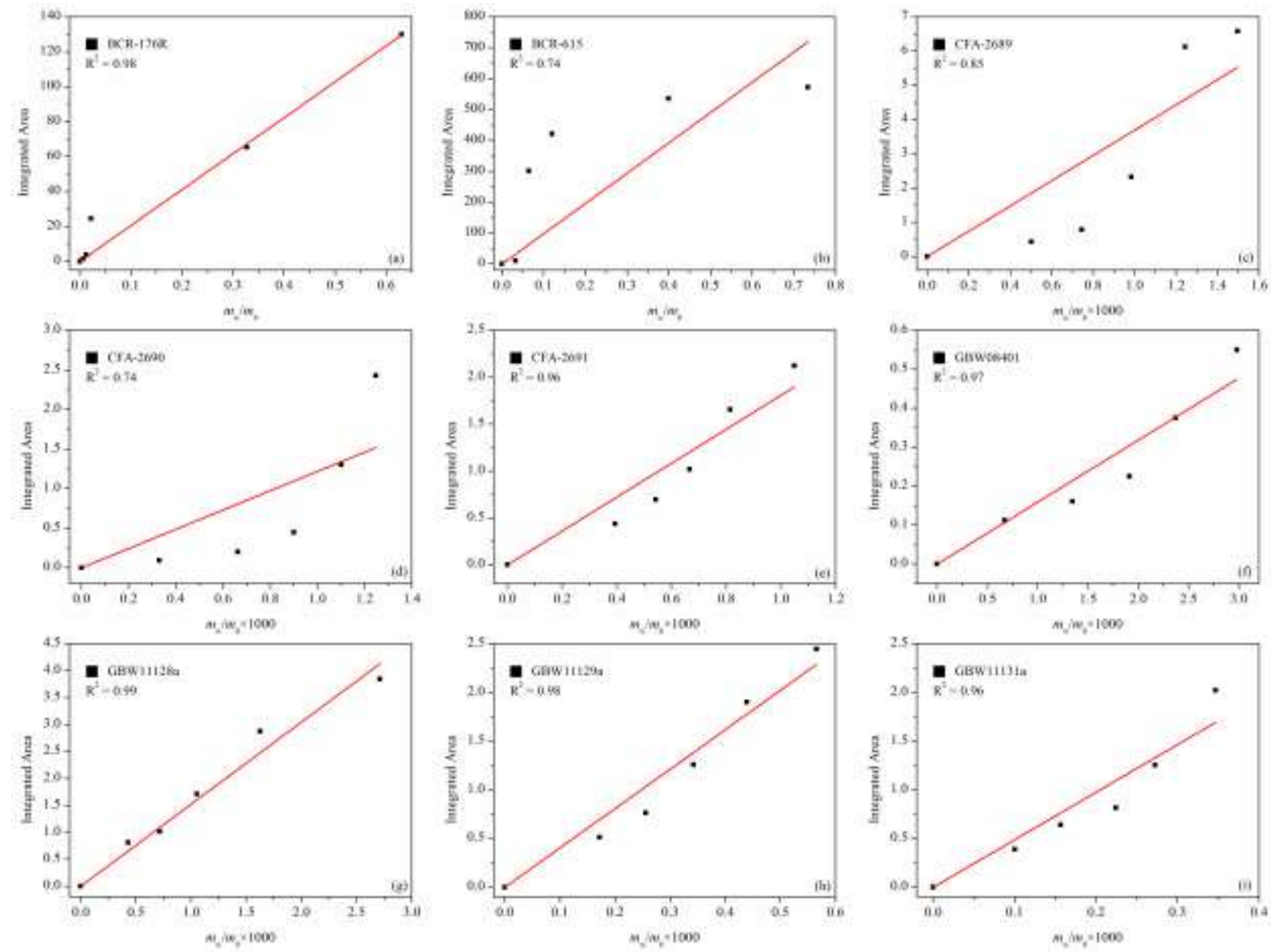

Figure S4. Integrated areas of IR peaks assigned to adsorbed water versus the mass ratios of adsorbed water to dry samples $\left(m_{\mathrm{w}} / m_{0}\right)$ for (a) BCR-176R, (b) BCR-615, (c) CFA-2689, (d) CFA2690, (e) CFA-2691, (f) GBW08401, (g) GBW11128a, (h) GBW11129a and (i) GBW11131a. 